\title{
Unemployment and Health-Related Quality of Life in Melanoma Patients During the COVID-19 Pandemic
}

\author{
Yeye Guo 1,2,3,4, Minxue Shen 1,2,3,4,5, Xu Zhang 1,2,3,4, Yi Xiao 1,2,3,4, Shuang Zhao 1,2,3,4, \\ Mingzhu Yin ${ }^{1,2,3,4}$, Wenbo Bu ${ }^{6}$, Yan Wang ${ }^{6}$, Xiang Chen ${ }^{1,2,3,4 *}$ and Juan Su ${ }^{1,2,3,4 *}$ \\ ${ }^{1}$ Department of Dermatology, Xiangya Hospital, Central South University, Changsha, China, ${ }^{2}$ National Clinical Research \\ Center for Geriatric Disorders, Xiangya Hospital, Changsha, China, ${ }^{3}$ Hunan Engineering Research Center of Skin Health and \\ Disease, Changsha, China, ${ }^{4}$ Hunan Key Laboratory of Skin Cancer and Psoriasis, Xiangya Hospital, Central South University, \\ Changsha, China, ${ }^{5}$ Department of Social Medicine and Health Management, Xiangya School of Public Health, Central South \\ University, Changsha, China, ${ }^{6}$ Jiangsu Key Laboratory of Molecular Biology for Skin Diseases and STIs, Institute of \\ Dermatology, Chinese Academy of Medical Science \& Peking Union Medical College, Nanjing, China
}

OPEN ACCESS

Edited by:

Enamul Kabir,

University of Southern

Queensland, Australia

Reviewed by:

Mohammad Hoque,

Agency for Clinical

Innovation, Australia

Márta Péntek,

Óbuda University, Hungary

*Correspondence:

Juan Su

sujuanderm@csu.edu.cn

Xiang Chen

chenxiangck@126.com

†These authors have contributed equally to this work

Specialty section: This article was submitted to Health Economics,

a section of the journal

Frontiers in Public Health

Received: 20 November 2020 Accepted: 03 February 2021

Published: 22 February 2021

Citation:

Guo $Y$, Shen $M$, Zhang $X$, Xiao $Y$, Zhao S, Yin M, Bu W, Wang Y, Chen $X$ and Su J (2021) Unemployment and Health-Related Quality of Life in

Melanoma Patients During the COVID-19 Pandemic.

Front. Public Health 9:630620. doi: 10.3389/fpubh.2021.630620
The outbreak of coronavirus disease-2019 (COVID-19) ineluctably caused social distancing and unemployment, which may bring additional health risks for patients with cancer. To investigate the association of the pandemic-related impacts with the health-related quality of life (HRQoL) among patients with melanoma during the COVID-19 pandemic, we conducted a cross-sectional study among Chinese patients with melanoma. A self-administered online questionnaire was distributed to melanoma patients through social media. Demographic and clinical data, and pandemic-related impacts (unemployment and income loss) were collected. HRQoL was determined by the Functional Assessment of Cancer Therapy-General (FACT-G) and its disease-specific module (the melanoma subscale, MS). A total of 135 patients with melanoma completed the study. The mean age of the patients was $55.8 \pm 14.2$ years, 48.1\% (65/135) were male, and $17.04 \%$ (34/135) were unemployed since the epidemic. Unemployment of the patients and their family members and income loss were significantly associated with a lower FACT-G score, while the MS score was associated with the unemployment of the patients' family members. Our findings suggested that unemployment is associated with impaired HRQoL in melanoma patients during the COVID-19 epidemic.

\section{Keywords: melanoma, coronavirus disease 2019 , unemployment, quality of life, income loss}

\section{INTRODUCTION}

The outbreak and pandemic of the coronavirus disease 2019 (COVID-19) brought profound impacts on the entire society and the individual's life. Besides the confirmed and suspected cases in hospitals or health facilities, the majority of people started the self-isolation at home voluntarily. However, the lockdown resulted in income loss and unemployment in some people. Under the circumstances, patients with cancers or chronic conditions may face higher risks of job loss and more mental and physical stress.

Melanoma is the most serious type of skin cancers with a highly aggressive ability. The 5 -years survival rate can be low as $5 \%$ for advanced melanoma (1), and the median overall survival time is about 8 months (2). Such poor survival outcomes result in heavy mental stress and impaired quality 
of life. Approximately one-third of patients with melanoma reported psychological distress to some extents, and symptoms of anxiety was more prevalent than depression (3). Neoadjuvant therapy such as checkpoint therapy and target therapy has been rapidly developed in recent years, and significantly benefited melanoma patients. However, high medical costs imposed heavy financial burdens on these patients. It was estimated that over 25,000 US dollars were cost per person annually for patients in late stages (4). As a result, unemployment and income loss may not only result in emotional problems but also lead to unaffordability during the advanced treatment for melanoma.

In the current study, we investigated the association of unemployment, income loss and other epidemic-related impacts with the health-related quality of life (HRQoL) in melanoma patients in China, based on an online questionnaire survey.

\section{MATERIALS AND METHODS \\ Study Design and Participants}

This was a cross-sectional study among Chinese patients with melanoma. We created an online survey link to facilitate the collection of questionnaires, and distributed it on social media (WeChat groups and teledermatology platforms where melanoma patients could communicate with other patients and doctors). Every single IP address was allowed only one entry and submission in order to avoid repeated submissions by individual patients. The final submission required the patients to complete all the questions. A post hoc analysis for sample size were performed through SAS Macro. The means and standard deviations of FACT-G were $77.53 \pm 15.39$ in the unaffected group $(N=58)$ and $64.26 \pm 18.15$ in the unemployed group $(N=23)$. According to the method of sample size estimation for Satterthwaite $t$-test proposed by Moser (5), assuming a double-sided significance level of 0.05 , a power of $80 \%$, and a sample ratio of $2: 1$, the minimum sample sizes will be 44 and 22, respectively, for each group. The survey was conducted between 4 Apr, 2020 and 15 Jan, 2021. The study was reviewed and approved by the institutional research ethics boards of Xiangya Hospital, Central South University (approval number: 202002024). Electronic informed consent was collected from all participants before the survey.

\section{Exposure}

The employment status was determined by a single question "What is your employment status since the epidemic of COVID19?" with the following three responses: "I am unemployed since the epidemic," "My employment status is unaffected since the epidemic", and "I was unemployed or retired before the epidemic." The employment status of the respondent's family members was also inquired in a similar way.

Income change was determined by a single question "Since the epidemic of COVID-19, is there any change in your monthly income?" with the following responses: "Complete income loss," "Income reduced," "Income unaffected (including the situation that I had no income before the epidemic)," and "income increased." Because only one patient reported increased income, this patient was also categorized as the "unaffected" group in the analysis.

Outdoor activity restriction was measured by a single question "During the past 2 weeks, how has your outdoor activity been affected?" with the following four responses: "My outdoor activity was unaffected," "My outdoor activity was partly restricted," and "I was isolated at home and receiving medical observation," "I was quarantined in hospital and receiving medical observation or treatment." Those who were isolated at home or in hospital were combined as one group.

\section{Outcome}

Functional Assessment of Cancer Therapy-Melanoma (FACT$\mathrm{M})$ was developed to evaluate the quality of life in melanoma patients (www.facit.org). FACT-M is an outcome measurement system composed of a core questionnaire for cancer patients, the FACT-General (FACT-G), and a disease-specific module (6). The questionnaire comprises of six subscales: physical well-being (PWB), social/family well-being (SWB), emotional well-being (EWB), functional well-being (FWB), melanoma subscale (MS), and melanoma surgery scale (MSS). The first four subscales belong to the FACT-G and the last two subscales are melanomaspecific elements. In our study, the primary outcome was the FACT-G score (the sum of PWB, SWB, EWB, and FWB subscales), and the secondary outcome was the MS score. The possible score ranges of the FACT-G and MS were 0-108 and 064, respectively, and higher scores indicate better HRQoL. The MSS was not analyzed since not all patients received surgery. The use of the FACT-M was authorized, and the Chinese version was obtained from the copyright holder before the survey.

\section{Co-variates}

Demographic and clinical information including gender, age, educational level (primary school and below, middle school, high school, college, graduate school), annual income (Chinese yuan, $\mathrm{CNY}$ ), marital status (unmarried, married, divorced, widowed), clinical stage of melanoma (I, II, III, IV), course of melanoma ( $<1$ year, 1-2 years, $\geq 3$ years, site of melanoma (extremities, trunk, head and neck, mucosal, unknown, other), current status of disease (stable, recurrent, metastasis), comorbidities (hypertension, diabetes, coronary heart disease, gout, etc.), adherence to treatment (no treatment prescribed, adherent to treatment, not adherent to treatment), and healthcare utilization (hospital visit, telemedicine) were collected and analyzed as covariates. For educational level, college and graduate school were combined as one group. Divorcement was not reported and therefore was not presented in the result. Because most patients had acral melanoma, all other sites were combined.

More details of the questionnaire are shown in Supplementary Data.

\section{Statistical Analyses}

There was no missing data in the questionnaire survey. The data were exported from the online survey system and analyzed with $\mathrm{R}$ version 3.5.2. Continuous variables with normal distribution were expressed as mean \pm standard deviation (SD) 
TABLE 1 | Demographic and clinical characteristics of the participants.

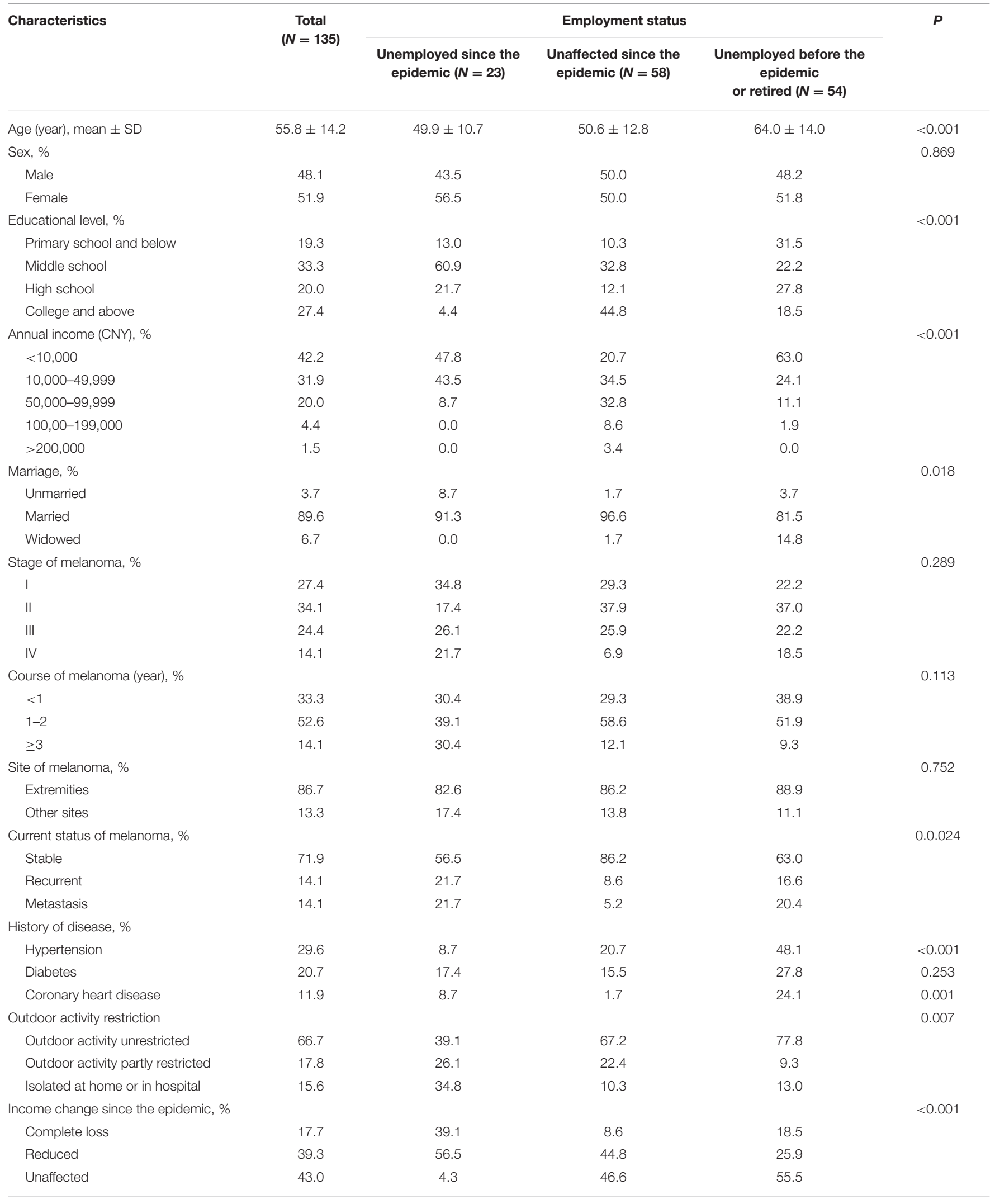

(Continued) 
TABLE 1 | Continued

\begin{tabular}{|c|c|c|c|c|c|}
\hline Characteristics & $\begin{array}{c}\text { Total } \\
(N=135)\end{array}$ & \multicolumn{3}{|c|}{ Employment status } & $P$ \\
\hline Unemployment of family members, \% & & & & & $<0.001$ \\
\hline Yes & 33.3 & 73.9 & 19.0 & 31.5 & \\
\hline No treatment prescribed & 48.1 & 30.4 & 53.4 & 50.0 & \\
\hline Not adherent to the treatment & 23.7 & 34.8 & 15.5 & 27.8 & \\
\hline Adherent to the treatment & 28.1 & 34.8 & 31.0 & 22.2 & \\
\hline \multicolumn{6}{|l|}{ Healthcare utilization } \\
\hline Visited a doctor in hospital, \% & 31.1 & 34.8 & 27.6 & 33.3 & 0.738 \\
\hline
\end{tabular}

and compared with analysis of variance (ANOVA). Hotelling's $\mathrm{T} 2$ test was used for the multivariate comparison of multiple subscale scores. Continuous data with skewed distribution were presented as median (interquartile range, IQR) and compared with Wilcoxon rank sum test. Categorical variables were summarized as counts (percentages) and compared using the chi-square test or Fisher's exact test. Stepwise linear regression with the corrected Akaike information criteria was used to identify variables that were associated with the outcomes. The effect size of the association was presented as regression coefficient and $95 \%$ confidence interval. $P<0.05$ was considered statistically significant. Reporting of the results followed the STROBE guideline.

\section{RESULTS}

From April 4, 2020 to Apr 11, 2020, a total of 135 valid questionnaires was collected and analyzed. None reported confirmed infection with COVID-19. The mean age of the patients was $55.8 \pm 14.2$ years, and 65 (48.1\%) were male. The characteristics of participants by employment status were shown in Table 1. Educational level, marital status, history of hypertension, income change since the epidemic, and employment status of family members were significantly different across the groups. The intracluster correlation coefficients of the subscales of FACT-M varied from 0.82 to 0.92 , indicating good internal reliability. The mean FACT-G and MS scores were 72.1 \pm 17.1 and $52.5 \pm 7.6$, respectively.

The distribution of the FACT-G and MS scores by employment status are shown in Figure 1A, with a clear positive correlation between the two scores. According to the Hotelling's Trace for multivariate test, unemployment was significantly associated with the PWB, SWB, and FWB subscale scores, but was not associated with the EWB and MS subscale scores (Figure 1B). The FACT-G score was the lowest in patients who were unemployed since the epidemic $(P=0.001)$; in contrast, the difference was not significant between the patients who were unaffected and those who were unemployed or retired before the epidemic (Figure 1C). Income loss and unemployment of family members were also significantly associated with lower FACT-G but not MS score, while adherence to treatment and isolation status were not associated with both outcomes (Figure 2). It is noteworthy that the patients who were unemployment during the epidemic had a lower proportion of immune therapy at present $(20.0 \%)$ compared to those whose income were unaffected $(70.6 \%)$.

The stepwise linear regression identified lower educational level, unemployment since the epidemic, and progression of melanoma as risk factors for impaired HRQoL measured as the FACT-G (Table 2). The progression of melanoma and unemployment of family members were significantly associated with the MS score.

\section{DISCUSSION}

In this study, we investigated the pandemic-related impacts on the HRQoL in melanoma patients through an online survey. The impaired HRQoL of melanoma was associated with the unemployment of the patients and their family members, as well as income loss. This is the first impact analysis of the COVID-19 for melanoma patients. These findings provide new insights into the health inequity issue arisen in the particular period.

Over 95 million people were documented to be infected with COVID-19 according to the World Health Organization (www.who.int). Besides the diseases, the pandemic led to a series social problems including reduced job opportunities. As reported by the United States Bureau of Labor Statistics, the unemployment rate was $12 \%$ in July 2020 , which exhibited the profound influence of the pandemic on the social economy. In the current study, we found that $17.04 \%$ of the melanoma patients were unemployed since the epidemic of COVID-19 and their HRQoL were decreased notably. It is well-established that the health status and psychological characteristics of patients are 
A

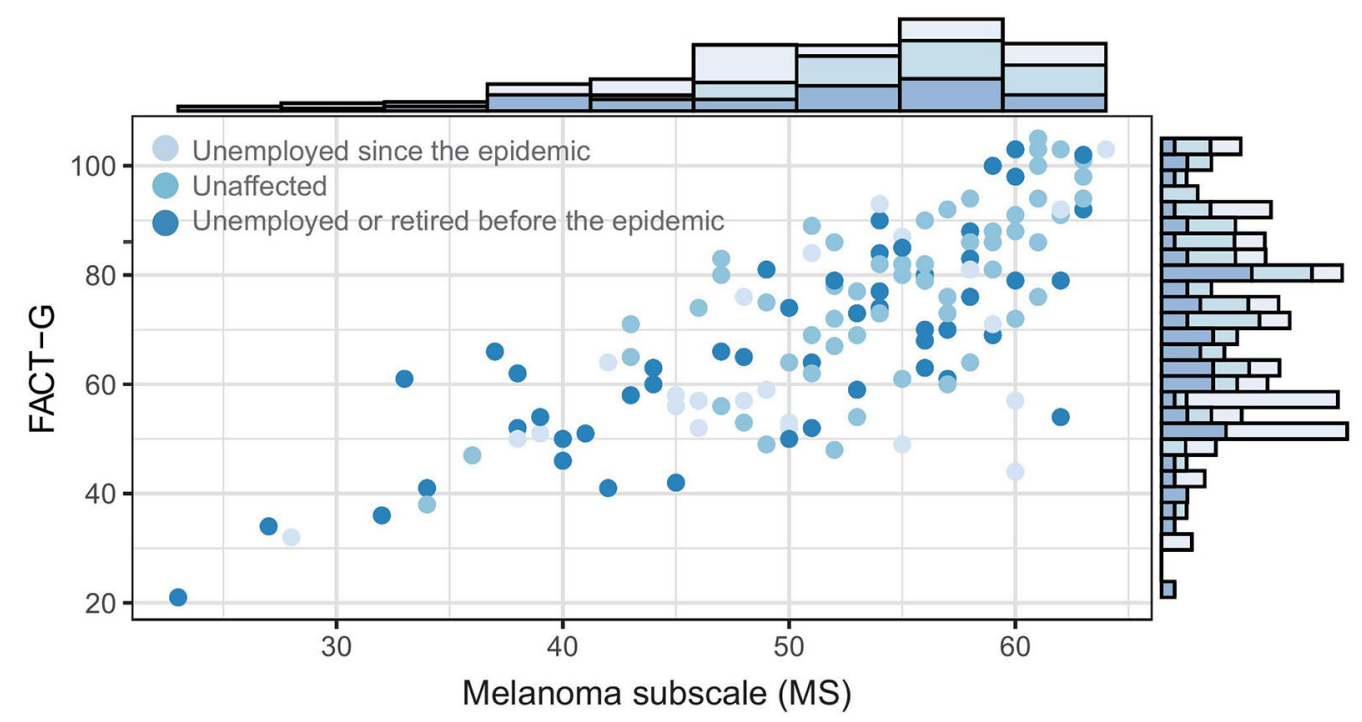

B

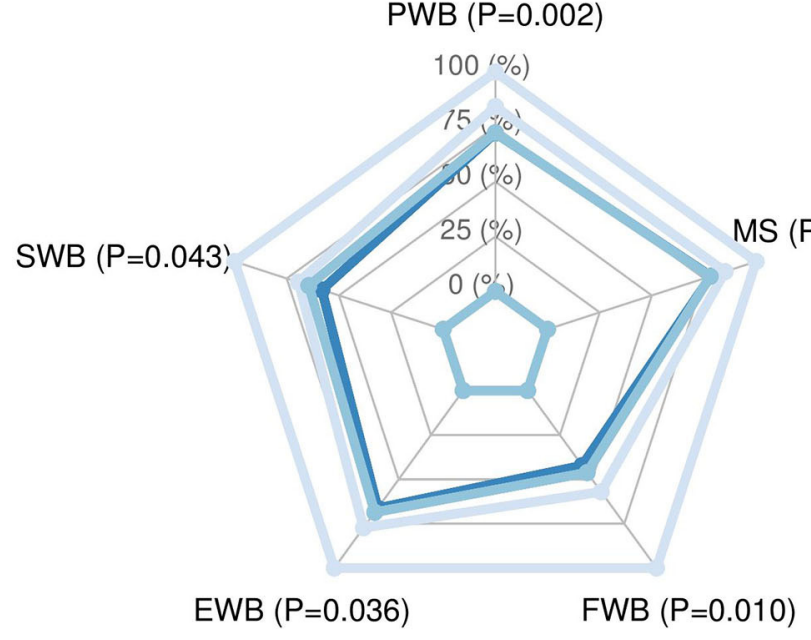

Unemployed since the epidemic

Unaffected

Unemployed or retired before the epidemic
C

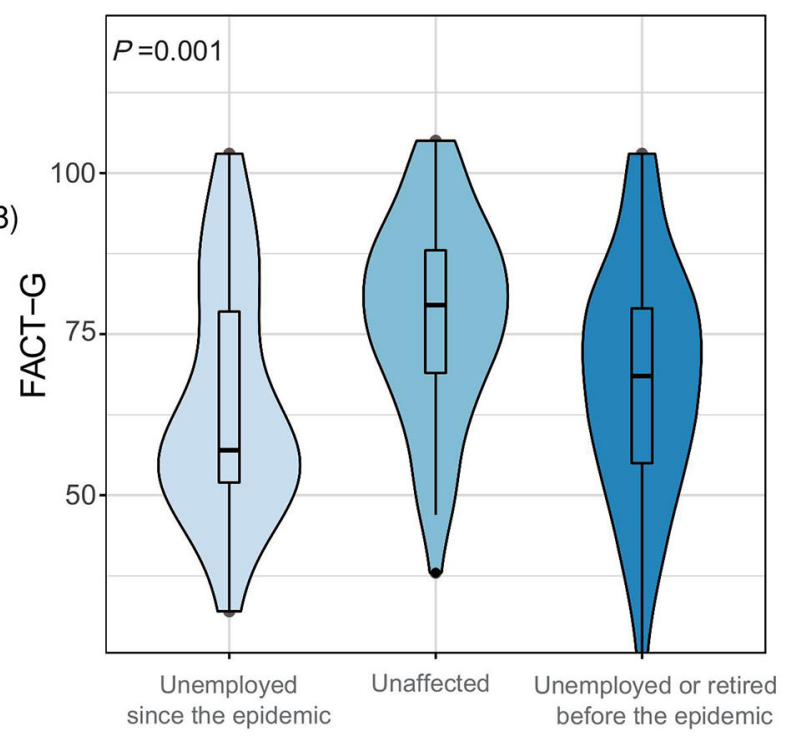

FIGURE 1 | Association of unemployment with health-related quality of life in melanoma patients. (A) Scatter plot between FACT-G and MS scores by the employment status. (B) Mean subscale scores of FACT-G by the employment status. (C) Violin-boxplot for the distribution of FACT-G score by the employment status. FACT-G, Functional Assessment of Cancer Therapy-General; MS, melanoma subscale; PWB, physical well-being; SWB, social/family well-being; EWB, emotional well-being; FWB, functional well-being.

associated with HRQoL. Previous studies found that patients with melanoma perceived persistent worries about developing new or metastatic cancers (7). A pervasive sense of uncertainty increases psychological distress, and impairs quality of life (8). Investigators found that systemic therapies can decrease HRQoL in a short time; however, the long-term HRQoL of melanoma survivors is comparable with the general population $(9,10)$. Interestingly, unemployment and a history of melanoma were previously identified to be associated with greater cancer worry, which indicated the importance of both melanoma and unemployment on psychological status (11). Our findings further emphasize the need to track the mental health in cancer patients under unemployment.

It has been estimated that over 100,000 people will be diagnosed with melanoma this year in the United States according to American Society of Clinical Oncology (ASCO). The incidence rate of melanoma in China was estimated at 0.9 per 100,000 , with a $110.3 \%$ rise compared to the 1990 s 

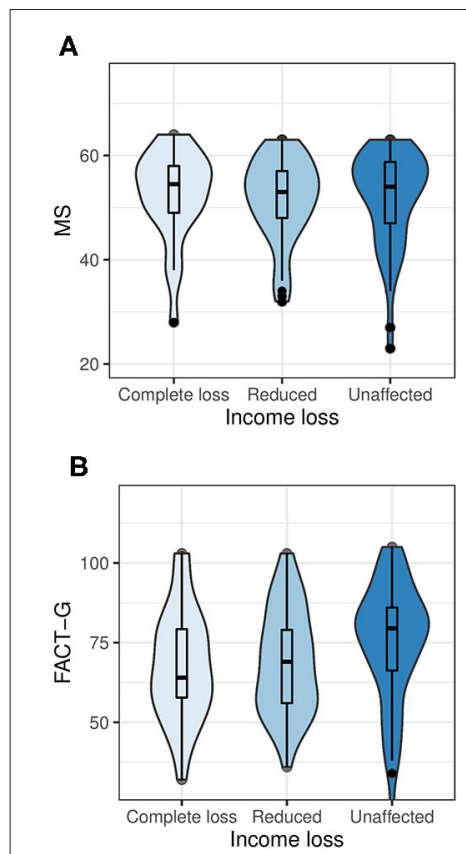

c

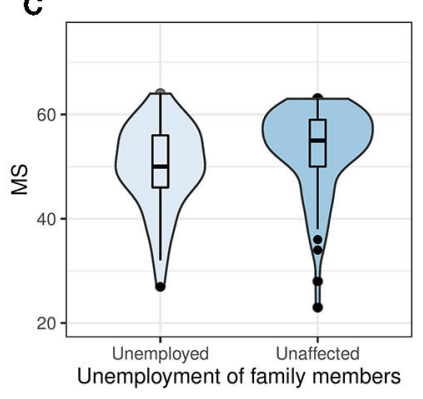

D

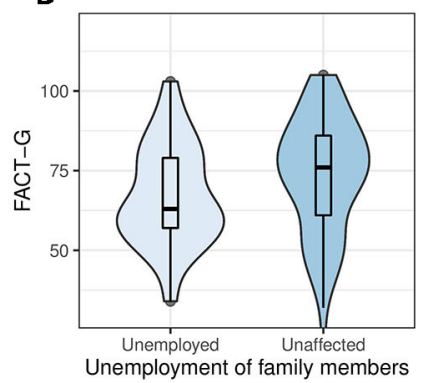

E

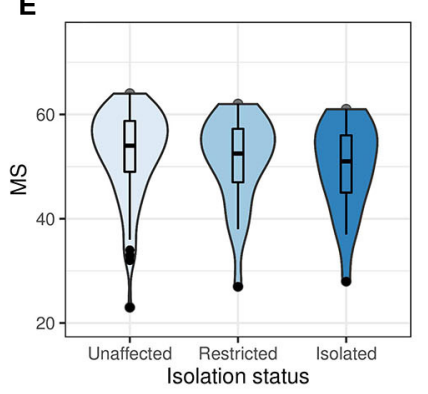

$F$

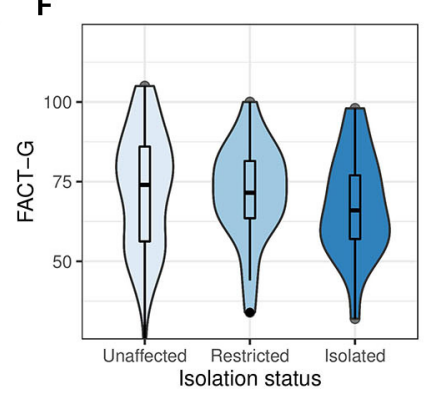

G

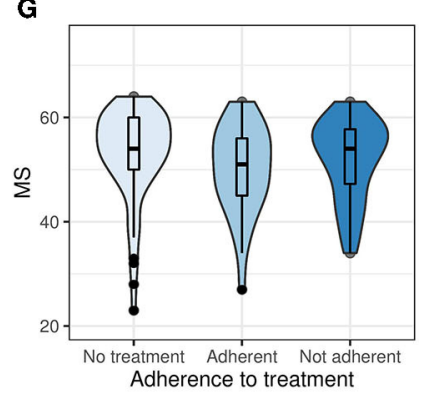

H

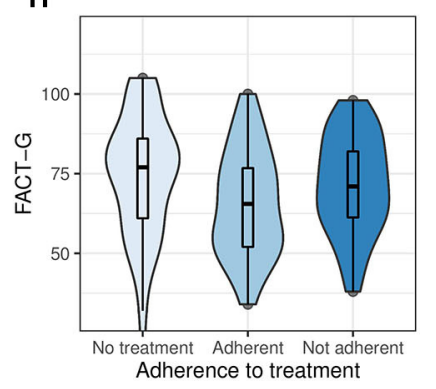

FIGURE 2 | Violin-boxplot for the distribution of the FACT-G and MS scores by income loss (A,B), unemployment of family members (C,D), isolation status (E,F), and adherence to treatment $\mathbf{( G , H )}$.

TABLE 2 | Stepwise linear regression for the FACT-G and MS scores.

\begin{tabular}{llcc}
\hline Outcomes Selected factors & Coefficient (SE) & $\boldsymbol{P}$ \\
\hline FACT-G & Education & & \\
& Primary school and below & Reference & \\
Middle school & $4.9(3.6)$ & 0.173 \\
High school & $14.6(3.9)$ & $<0.001$ \\
College and above & $14.0(3.8)$ & $<0.001$ \\
Employment status & & \\
Unemployed since the epidemic & $-14.9(3.6)$ & $<0.001$ \\
Unaffected & Reference & \\
Unemployed before the epidemic or retired & $-3.3(2.9)$ & 0.259 \\
Current status of melanoma, $n(\%)$ & & \\
Stable & Reference & \\
Recurrent & $-10.9(3.6)$ & 0.003 \\
Metastasis & $-24.8(3.6)$ & $<0.001$ \\
Current status of melanoma, $n(\%)$ & & \\
Stable & Reference & \\
Recurrent & $-5.0(1.7)$ & 0.003 \\
Metastasis & $-14.6(1.7)$ & $<0.001$ \\
Unemployment of family members & & \\
No & Reference & \\
Yes & $-4.3(1.2)$ & $<0.001$ \\
\hline MS & & \\
\hline
\end{tabular}

SE, standard error. FACT-G, Functional Assessment of Cancer Therapy-General, MS, melanoma subscale.

(12). The increasing incidence of melanoma makes it a public health concern and brings the global burden of disease (13). The disability-adjusted life-years (DALYs) of melanoma have been increasing in China during the last decade, while the DALYs of common cancers such as esophageal cancer and stomach cancer have decreased significantly (12). In recent years, the development of immune therapy benefits cancer patients substantially, but also leads to a heavy economic burden for them (14). A review indicates that higher spending on cancer is consistently associated with lower mortality (15). In other words, loss of income and work-related benefits experienced by the unemployed may lead to adverse health outcomes (16). Unemployment of the family members further impairs the social support and financial source, resulting in unaffordability of the advanced therapies and, the progression of cancer and mental stress. This is supported by our finding that unemployment of family member was an independent risk factor for lower MS score. Additionally, the proportion of the use of immune therapy in melanoma patients with unemployment was only one-third of those who did not experience the unemployment. Considering the highly cost of immune therapy, which costs around 4000 USD monthly, the result indicates that financial status might be an essential consideration in the selection of treatment strategy. These findings indicate that the health disparities in cancer could result in a vicious circle. Social setting, incorporating poverty, culture, and social justice were found to play a part in disease outcome (17). Some poverty-related barriers such as income, education, and health insurance could influence melanoma outcomes (18). This makes social justice quite essential to diminish the disparities in melanoma.

To the best of our knowledge, this is the first empirical study of pandemic-related impacts on the HRQoL of melanoma patients. Our finding indicates that early and timely mental health 
intervention, telemedicine, and health education are needed for melanoma patients. More importantly, health decision makers should consider the reimbursement policy of advanced treatment methods for cancers with high fatality rate.

\section{Limitation}

There are limitations in our study. First, we conducted an online survey instead of in-person interview because of the high infectivity of the COVID-19. Therefore, the representativeness of our sample might be limited owing to selection bias. Second, a total of 135 patients participated in the study despite our efforts to recruit as more patients as we could. The sample size is relatively small which may lead to insufficient power of test to identify differences. Third, the exposure and outcome variables were self-reported, and recall bias might be introduced. Last, the survey was conducted among Chinese patients, which may not fully represent melanoma patients beyond China owing to the differences in culture, reimbursement policy, and social system.

\section{DATA AVAILABILITY STATEMENT}

The raw data supporting the conclusions of this article will be made available by the authors, without undue reservation.

\section{ETHICS STATEMENT}

The studies involving human participants were reviewed and approved by the institutional research ethics boards of Xiangya Hospital, Central South University (approval number: 202002024). The patients/participants provided their written informed consent to participate in this study.

\section{REFERENCES}

1. Topalian SL, Hodi FS, Brahmer JR, Gettinger SN, Smith DC, McDermott DF, et al. Five-year survival and correlates among patients with advanced melanoma, renal cell carcinoma, or nonsmall cell lung cancer treated with nivolumab. JAMA Oncol. (2019) 5:1411-20. doi: 10.1001/jamaoncol.2019.2187

2. Miller AJ, Mihm MCJr. Melanoma. N Engl J Med. (2006) 355:5165. doi: 10.1056/NEJMra052166

3. Kasparian NA, McLoone JK, Butow PN. Psychological responses and coping strategies among patients with malignant melanoma: a systematic review of the literature. Arch Dermatol. (2009) 145:1415-27. doi: 10.1001/archdermatol.2009.308

4. Seidler AM, Pennie ML, Veledar E, Culler SD, Chen SC. Economic burden of melanoma in the elderly population: population-based analysis of the Surveillance, Epidemiology, and End Results (SEER)-Medicare data. Arch Dermatol. (2010) 146:249-56. doi: 10.1001/archdermatol.2009.389

5. Moser BK, Stevens GR, Watts CL. The two-sample t test versus satterthwaite's approximate f test. Commun Stat Theor Methods. (1989) 18:3963-75. doi: 10.1080/03610928908830135

6. Cormier JN, Ross MI, Gershenwald JE, Lee JE, Mansfield PF, Camacho $\mathrm{LH}$, et al. Prospective assessment of the reliability, validity, and sensitivity to change of the Functional Assessment of Cancer TherapyMelanoma questionnaire. Cancer. (2008) 112:2249-57. doi: 10.1002/cncr. 23424

\section{AUTHOR CONTRIBUTIONS}

$\mathrm{XC}$ and JS: conception and design. MS: administrative support. JS, SZ, MY, WB, and YW: provision of study materials or patients. YG, MS, and XZ: collection and assembly of data. YG, MS, and YX: data analysis and interpretation. All authors manuscript writing and final approval of manuscript.

\section{FUNDING}

This work was supported by the National Natural Science Foundation of China (62041208, 81974478), the Ministry of Science and Technology of the People's Republic of China (2016YFC0900802), the Emergency Project of Prevention and Control for COVID-19 of Central South University (502701002), the Department of Science and Technology of Hunan Province (2018SK2086, 2018SK2092), and China Scholarship Council (201906370201).

\section{ACKNOWLEDGMENTS}

We would like to thank all the melanoma patients who participated in our study for their support and assistance in the online survey. Particularly, we want to express our high regard to all the health practitioners and volunteers for their dedication in the fight against COVID-19.

\section{SUPPLEMENTARY MATERIAL}

The Supplementary Material for this article can be found online at: https://www.frontiersin.org/articles/10.3389/fpubh. 2021.630620/full\#supplementary-material

7. McLoone J, Watts K, Menzies S, Meiser B, Butow P, Kasparian N. When the risks are high: psychological adjustment among melanoma survivors at high risk of developing new primary disease. Qual Health Res. (2012) 22:1102-13. doi: 10.1177/1049732312448542

8. Johnson Wright L, Afari N, Zautra A. The illness uncertainty concept: a review. Curr Pain Headache Rep. (2009) 13:1338. doi: 10.1007/s11916-009-0023-z

9. Cornish D, Holterhues C, van de Poll-Franse LV, Coebergh JW, Nijsten T. A systematic review of health-related quality of life in cutaneous melanoma. Ann Oncol. (2009) 20 (Suppl. 6):vi51-58. doi: 10.1093/annonc/mdp255

10. Heino PJ, Myllari PH, Jahkola TA, Sintonen H, Luoma ML, Rasanen $\mathrm{P}$, et al. Long-term quality of life of melanoma survivors is comparable to that of the general population. Anticancer Res. (2019) 39:263340. doi: 10.21873/anticanres.13387

11. Khoshab N, Vaidya TS, Dusza S, Nehal KS, Lee EH. Factors contributing to cancer worry in the skin cancer population. J Am Acad Dermatol. (2019) 179:88-94. doi: 10.1016/j.jaad.2019.09.068

12. Wu Y, Wang Y, Wang L, Yin P, Lin Y, Zhou M. Burden of melanoma in China, 1990-2017: findings from the 2017 global burden of disease study. Int J Cancer. (2020) 147:692-701. doi: 10.1002/ijc.32764

13. Karimkhani C, Green AC, Nijsten T, Weinstock MA, Dellavalle RP, Naghavi $\mathrm{M}$, et al. The global burden of melanoma: results from the Global Burden of Disease Study 2015. Br J Dermatol. (2017) 177:134-40. doi: 10.1111/bjd.15510

14. Verma V, Sprave T, Haque W, Simone II, Chang JY, Welsh JW, et al. A systematic review of the cost and cost-effectiveness studies 
of immune checkpoint inhibitors. J Immunother Cancer(2018) 6:128. doi: 10.1186/s40425-018-0442-7

15. Li M, Lakdawalla DN, Goldman DP. Association between spending and outcomes for patients with cancer. J Clin Oncol. (2020) 38:32331. doi: 10.1200/JCO.19.01451

16. Stauder J. Unemployment, unemployment duration, and health: selection or causation? Eur J Health Econ. (2019) 20:5973. doi: 10.1007/s10198-018-0982-2

17. Freeman HP, Chu KC. Determinants of cancer disparities: barriers to cancer screening, diagnosis, and treatment. Surg Oncol Clin N Am. (2005) 14:655-69, v. doi: 10.1016/j.soc.2005. 06.002

18. Harvey VM, Patel H, Sandhu S, Wallington SF, Hinds G. Social determinants of racial and ethnic disparities in cutaneous melanoma outcomes. Cancer Control. (2014) 21:343-9. doi: 10.1177/107327481402 100411

Conflict of Interest: The authors declare that the research was conducted in the absence of any commercial or financial relationships that could be construed as a potential conflict of interest.

Copyright (c) 2021 Guo, Shen, Zhang, Xiao, Zhao, Yin, Bu, Wang, Chen and Su. This is an open-access article distributed under the terms of the Creative Commons Attribution License (CC BY). The use, distribution or reproduction in other forums is permitted, provided the original author(s) and the copyright owner(s) are credited and that the original publication in this journal is cited, in accordance with accepted academic practice. No use, distribution or reproduction is permitted which does not comply with these terms. 